\title{
Vortex particle-mesh simulations of vertical axis wind turbine flows: from the airfoil performance to the very far wake
}

\author{
Philippe Chatelain $^{1}$, Matthieu Duponcheel ${ }^{1}$, Denis-Gabriel Caprace ${ }^{1}$, Yves Marichal ${ }^{1,2}$, and \\ Grégoire Winckelmans ${ }^{1}$ \\ ${ }^{1}$ Institute of Mechanics, Materials and Civil Engineering, Université catholique de Louvain, \\ 1348 Louvain-la-Neuve, Belgium \\ ${ }^{2}$ Wake Prediction Technologies (WaPT), Rue Louis de Geer 6, 1348 Louvain-la-Neuve, Belgium \\ Correspondence to: Philippe Chatelain (philippe.chatelain@uclouvain.be)
}

Received: 15 December 2016 - Discussion started: 2 January 2017

Revised: 10 April 2017 - Accepted: 16 May 2017 - Published: 19 June 2017

\begin{abstract}
A vortex particle-mesh (VPM) method with immersed lifting lines has been developed and validated. Based on the vorticity-velocity formulation of the Navier-Stokes equations, it combines the advantages of a particle method and of a mesh-based approach. The immersed lifting lines handle the creation of vorticity from the blade elements and its early development. Large-eddy simulation (LES) of vertical axis wind turbine (VAWT) flows is performed. The complex wake development is captured in detail and over up to 15 diameters downstream: from the blades to the near-wake coherent vortices and then through the transitional ones to the fully developed turbulent far wake (beyond 10 rotor diameters). The statistics and topology of the mean flow are studied. The computational sizes also allow insights into the detailed unsteady vortex dynamics and topological flow features, such as a recirculation region influenced by the tip speed ratio and the rotor geometry.
\end{abstract}

\section{Introduction}

The aerodynamics of vertical axis wind turbines (VAWTs) are inherently unsteady, which leads to vorticity shedding mechanisms due to both the lift distribution along the blade and its time evolution. This translates into a wake topology that is far more complex and unsteady than for VAWTs' horizontal axis counterparts (HAWTs), a characteristic which could be indicative of more intense wake decay mechanisms for VAWTs. Additionally, their inherent insensitivity to wind direction changes suggests a more robust efficiency in turbulent conditions. Naturally, both traits have led to several claims of an advantage of VAWTs over HAWTs in wind farms (e.g., Paquette and Barone, 2012; Kinzel et al., 2012) and thus to promises of higher power extraction densities. These, together with potential operational gains (maintenance costs, the disappearance of yawing actuation), have led to some definite research momentum in VAWT aerodynamics, in the shape of experimental (Ferreira et al.,
2009; Beaudet, 2014) and numerical (Scheurich, 2011; Ferreira et al., 2014) studies. However, because of their unsteady aerodynamics, VAWT simulation and modeling tools have not reached yet the level of development of those for HAWTs, e.g., the blade element momentum method. Numerical investigations of VAWT wake phenomena have only been tackled recently (Scheurich and Brown, 2013), but the volume of these efforts is quite underwhelming when compared to all the comparable works on HAWTs (Sørensen et al., 2015), and the computational domains and resolutions of existing studies are quite limited. In this paper, we perform large-scale, highly resolved large-eddy simulation of the flows past vertical axis wind turbines by means of a state-of-the-art vortex particle-mesh (VPM) method combined with immersed lifting lines (Chatelain et al., 2013). We focus on the intrinsic vortex dynamics and wake decay mechanisms; all simulations are thus carried out without turbulence in the wind. The simulation tool is validated against experimental aerodynamic data and is then run for a standard, 
medium-solidity, H-shaped machine: mean flow and turbulence statistics are computed over more than 15 diameters downstream of the machine. The sensitivity of the wake behavior to the operating conditions (tip speed ratio, TSR) and to the machine aspect ratio (AR) is also assessed. This paper is structured as follows. We briefly call to mind the VPM method in Sect. 2 and present some of the advances that enabled the large-eddy simulation of wind turbines within this VPM context: the multiscale sub-grid scale model and the modeling of blades through immersed lifting lines. Section 3 presents some validation of our methodology and then moves to the study of a standard VAWT from the perspectives of its aerodynamics and its wake dynamics. We close this paper in Sect. 4 with our conclusions and perspectives.

\section{Methodology}

\subsection{The vortex particle-mesh method}

The coarse-scale aerodynamics and the wake of the VAWT are simulated using a massively parallel implementation of a vortex particle-mesh flow solver. The present method relies on the large-eddy simulation in the vorticity-velocity formulation for incompressible flows $(\nabla \cdot \boldsymbol{u}=0)$ :

$$
\frac{D \boldsymbol{\omega}}{D t}=(\boldsymbol{\omega} \cdot \nabla) \boldsymbol{u}+v \nabla^{2} \boldsymbol{\omega}+\nabla \cdot \mathbf{T}^{M},
$$

where $v$ is the kinematic viscosity and $\mathbf{T}^{M}$ is the sub-grid scale (SGS) model. The velocity field is recovered from the vorticity by solving the Poisson equation

$\nabla^{2} \boldsymbol{u}=-\nabla \times \omega$.

The advection of vorticity is handled in a Lagrangian fashion using particles, characterized by a position $\boldsymbol{x}_{\mathrm{p}}$, a volume $V_{\mathrm{p}}$, and a vorticity integral $\alpha_{\mathrm{p}}=\int_{V_{\mathrm{p}}} \omega \mathrm{d} \boldsymbol{x}$ :

$$
\begin{aligned}
\frac{\mathrm{d} \boldsymbol{x}_{\mathrm{p}}}{\mathrm{d} t} & =\boldsymbol{u}_{\mathrm{p}}, \\
\frac{\mathrm{d} \boldsymbol{\alpha}_{\mathrm{p}}}{\mathrm{d} t} & =\left((\boldsymbol{\omega} \cdot \nabla) \boldsymbol{u}+v \nabla^{2} \boldsymbol{\omega}+\nabla \cdot \mathbf{T}^{M}\right)_{\mathrm{p}} V_{\mathrm{p}},
\end{aligned}
$$

where we identify the roles of the velocity field in the advection and of the vortex stretching, diffusion and SGS terms for the evolution of vorticity.

The right-hand sides of these equations are evaluated efficiently on an underlying mesh (Chatelain et al., 2008). The stretching and diffusion operators use fourth-order finite differences, and Eq. (2) is solved efficiently with a Fourierbased solver. In this work, we rely on the technique used by Chatelain and Koumoutsakos (2010), which handled a combination of periodic and unbounded directions through the approach of Hockney and Eastwood (1988). It is here extended to an inflow-outflow direction, say $x$, and two unbounded directions, $y$ and $z$. A Fourier transform along $x$ yields

$\nabla_{y z}^{2} \widetilde{\boldsymbol{u}}-k_{x}^{2} \widetilde{\boldsymbol{u}}=-\widetilde{\nabla \times \omega}$,

where $\widetilde{\boldsymbol{u}}\left(k_{x}, y, z\right)$ stands for the $x$-transformed field. For a given $k_{x}$ mode, this is a two-dimensional Helmholtz equation in an unbounded $(y, z)$-domain; it is solved through a convolution with the corresponding Green's function in Fourier space through the domain-doubling technique of Hockney and Eastwood (1988); see Chatelain and Koumoutsakos (2010) for details. The wave numbers $k_{x}$ are here constrained to produce inflow-outflow conditions or equivalently to only permit adequately phased sine or cosine modes. The following conditions on the streamwise velocity are then imposed: $u_{x}(0, y, z)=U_{\infty}$ at the inflow and $\frac{\partial u_{x}}{\partial x}\left(L_{x}, y, z\right)=$ 0 at the outflow. These are completed with the conditions on the transverse components: $\frac{\partial u_{y}}{\partial x}(0, y, z)=\frac{\partial u_{z}}{\partial x}(0, y, z)=0$ and $u_{y}\left(L_{x}, y, z\right)=u_{z}\left(L_{x}, y, z\right)=0$.

The SGS model is a simplified version of the variational multiscale (VM) model (Hughes et al., 2001), known as the regularized version (RVM) (Jeanmart and Winckelmans, 2007). In that variant, the SGS model is designed as an eddy viscosity model acting only on the small-scale field

$\mathbf{T}^{M}=v_{\mathrm{SGS}}\left(\nabla \boldsymbol{\omega}^{\mathrm{s}}+\nabla^{t} \boldsymbol{\omega}^{\mathrm{s}}\right)$,

where $\omega^{\mathrm{s}}$ represents the small-scale part of the vorticity field obtained by high-pass filtering. The eddy viscosity is taken as $\nu_{\mathrm{SGS}}=C_{r}^{(n)} \Delta^{2}(2 \mathbf{S}: \mathbf{S})^{1 / 2}$, where the strain rate $\mathbf{S}$ is evaluated using the complete velocity field. We refer to Cocle et al. $(2007,2008,2009)$ for implementation details and for the values of the coefficients $C_{r}^{(n)}$ when using filtering of the order $2 n$.

In order to carry out the computational steps above, information is made available on the mesh, and recuperated from the mesh, by interpolating back and forth between the particles and the grid using high-order interpolation schemes. Fortunately, this hybridization does not affect the good numerical accuracy (in terms of diffusion and dispersion errors) and the stability properties of a particle method. The present method does indeed still waive the typical CourantFriedrichs-Lewy (CFL) constraint for the explicit time integration of advection, $\Delta t<C_{u} h /\|\boldsymbol{u}\|_{\max }$, and instead involves higher-order constraints (Koumoutsakos, 2005), e.g., $\Delta t<C_{\nabla u} /\|\nabla \boldsymbol{u}\|_{\max }$; this essentially corresponds to preventing particle trajectories from crossing each other. The time integration scheme used in the present work is a low-storage third-order Runge-Kutta (Williamson, 1980).

This last discussion actually pertains to the issue of Lagrangian distortion in particle methods. If left alone, particles can be seen to deplete regions of the flow or cluster in regions. Several remedies have been proposed. Dissipative terms can be added to the particle ordinary differential equations (ODEs) in order to limit the particle deformations (Monaghan, 2000); this comes at the price of 
artificial bulk and shear viscosities. State-of-the-art particle methods, such as the present one, rely on a procedure called remeshing (Cottet, 1996; Koumoutsakos, 1997; Ploumhans and Winckelmans, 2000; Winckelmans, 2004), which consists of the periodic regularization of the particle set onto a mesh. This procedure typically relies on high-order interpolation formulas (Monaghan, 1985; van Rees et al., 2011) which involve well-controlled levels of artificial viscosity. All the simulations of Sect. 3 have involved a remeshing operation every five time steps that uses the third-order accurate $M_{4}^{\prime}$ scheme of Monaghan (1985); the same scheme is used for the particle-to-mesh and mesh-to-particle interpolation operations.

\subsection{Immersed lifting lines}

The generation of vorticity along the blades is accounted for through an immersed lifting line approach (Chatelain et al., 2013). The approach is very much akin to a vortex lattice method and relies on the Kutta-Joukowski theorem (see, e.g., Prandtl, 1923) that relates the developed lift $\boldsymbol{L}$ to the relative flow $\boldsymbol{V}_{\text {rel }}$ and the circulation bound around the local 2-D airfoil

$\boldsymbol{L}=\rho \boldsymbol{V}_{\text {rel }} \times \boldsymbol{\Gamma}$.

Lift can also be obtained from the relative flow, its angle of attack $\alpha$ and the airfoil lift coefficient $C_{1}(\alpha)$; equating this aerodynamics-provided expression to Eq. (7) allows us to solve for the instantaneous circulation $\boldsymbol{\Gamma}$ at a blade location. The solenoidal property of vorticity then imposes that streamwise and spanwise vorticities be shed from the lifting line in order to account for spanwise and temporal variations of $\boldsymbol{\Gamma}$, respectively. Over a time step, the shed vorticity is constructed thanks to Lagrangian tracers released along the blade. The vorticity bound to the blade and the newly generated vorticity are discretized by means of particles immersed in the mesh and in the bulk-flow-representing particles; their treatment thus fits within the present particlemesh framework. Unlike the mesh-only Vorticity Transport Model (Brown and Line, 2002) or an actuator line technique (Sørensen et al., 2015), this treatment of vorticity sources is Lagrangian and well suited for the large time steps enabled by the rest of the method. The aerodynamic behavior of the lifting lines sections, i.e., $C_{1}$ and $\boldsymbol{\Gamma}$, account for unsteady effects through a Leishman-Beddoes dynamic stall model (Leishman, 2006). This semiempirical model shows a good trade-off between simplicity and accuracy, provided that the model coefficients are validated with relevant experimental data. In this work, we follow the indications of Dyachuk et al. (2014) and Scheurich (2011), who present coefficients for various airfoils validated in the particular case of a VAWT.

The standard lifting line and the actuator line techniques are not able to capture flow curvature effects. Indeed, if the flow relative to the blade is curved, as is the case here for a blade in rotation through essentially straight streamlines, the airfoil behaves as an airfoil with an additional camber (Migliore and Wolfe, 1979; Beaudet, 2014). We consider a blade with a chord $c$ tangentially positioned at a radius $R$ for its quarter-chord position; this additional camber can be modeled in a straightforward manner by pitching the blade inwards by an angle $\alpha_{0}=\arctan ((1-\cos (\beta / 2)) / \sin (\beta / 2))$ where $\beta=\arctan (c / 2 R)+\arctan (c / 2 R)$. In the validation section below (Sect. 3.1), we verify the positive effect of such a correction. Finally, we note that these methods (immersed lifting line or actuator line) in their standard versions do not capture the internal turbulent fluctuations of the structures actually shed.

\section{Results}

\subsection{Validation}

We first present validation results against recent work (Castelein, 2015) for a low-solidity two-bladed $\mathrm{H}$-shaped machine with NACA0018 airfoils. The parameters for the Leishman-Beddoes dynamic stall model are based on those for a NACA0015 in Scheurich (2011); they are here tuned to fit the static behavior of the polar at the Reynolds number of the experiment at the design point, $R e=U_{\text {rel }} c / v=1.5 \times 10^{5}$. Throughout this paper, we use the following axes convention: $x$ is the streamwise direction; $y$ is cross-stream and orthogonal to the VAWT axis, which $z$ is parallel to. The origin for the blade angular position $\theta$ is set to the position moving upwind. Figure 1 presents the profiles of the normal and tangential forces developed by a blade over a revolution, nondimensionalized with respect to the profile chord $c$ and the dynamic pressure $q_{0}=1 / 2 \rho U_{\infty}^{2}$.

We report on VPM simulations with and without a curvature correction, which here amounts to an inward pitch $\alpha_{0}=$ $1.72^{\circ}$. This correction appears to bring a notable improvement of the results, particularly for the moderate TSR $=$ $\Omega R / U_{\infty}$ : the explored angles of attack are indeed smaller than at low TSR. While the results at an intermediate TSR show good agreement (Fig. 1b), there is a clear departure at the lower TSR (Fig. 1a). The experimental points suggest a stall happening later on the upstream stretch, at around $90^{\circ}$, and more abruptly than for the simulation; we report here that the authors of the experiment advised circumspection when using the $F_{\mathrm{n}}$ data at $135^{\circ}$ and clearly question the validity of their results for $F_{\mathrm{t}}$ through the whole rotation. We, nevertheless, compare our simulations to all their results in Fig. 1. This mismatch in the upstream part has a direct influence on the predictions for the downstream stretch $\left(\theta \in\left[210,270^{\circ}\right]\right)$, as the stall-generated structures are advected through the rotor; this may explain the marked differences observed there. These results are satisfactory: they are indeed very sensitive to the dynamic stall model, here probably still misadapted, and to some unquantified uncertainties for the experimen- 

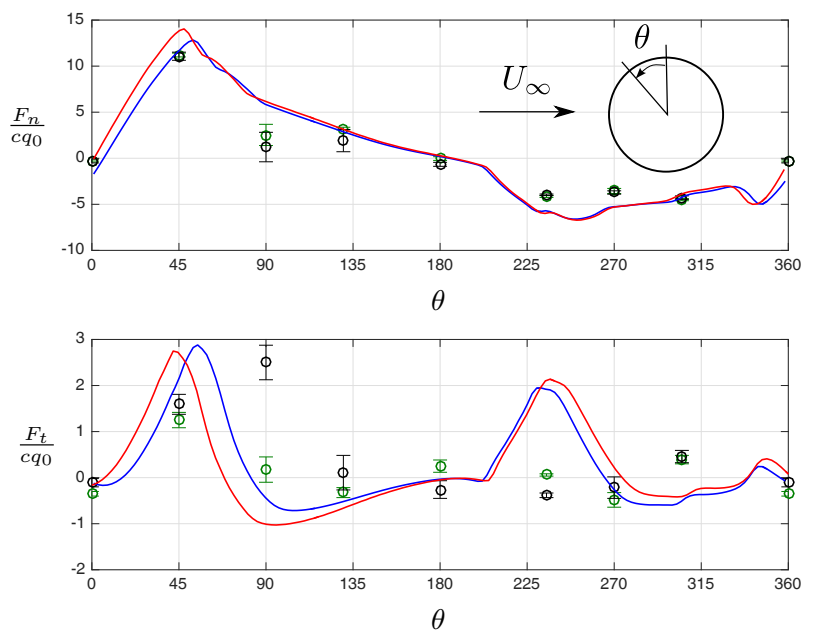

(a) $\operatorname{TSR}=2.0$
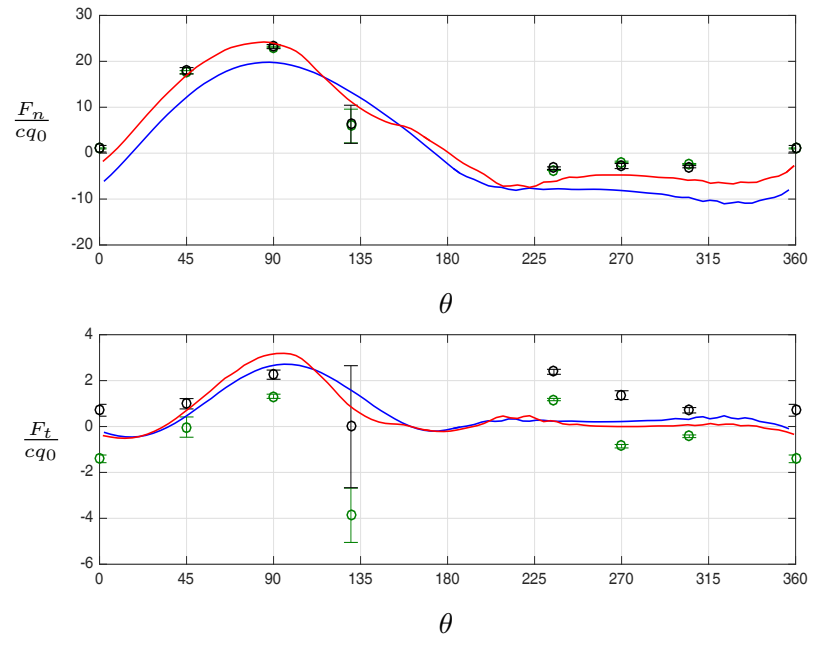

(b) $\mathrm{TSR}=4.5$

Figure 1. Validation: evolution of the normal, $F_{\mathrm{n}} /\left(c q_{0}\right)$, and tangential, $F_{\mathrm{t}} /\left(c q_{0}\right)$, force coefficients at mid-height vs. the blade angular position $\theta$; VPM simulation without curvature correction (blue solid line) and with curvature correction (red solid line); experimental results (o) with two techniques of force computation from particle image velocimetry (PIV) flow fields (Castelein, 2015).

tal facility (the TU Delft Open Jet Wind Tunnel), namely its blockage and secondary flows in the test section.

\subsection{Aerodynamics}

The remainder of this section focuses on a low-solidity HVAWT studied numerically by Scheurich et al. (2010) and Scheurich (2011). For the sake of completeness, we here briefly recall its main parameters: an aspect ratio $\mathrm{AR}=$ $H / D=1.5$, a solidity $\sigma=n c / D=0.1725$, and constantchord NACA0015 airfoils. Simulations were run without the curvature correction investigated above (or equivalently, the simulated corresponds to a machine with a blade pitched outwards by $\alpha_{0}=1.65^{\circ}$ ).

Figure 2 shows this machine's power coefficient $\left(C_{\mathrm{p}}\right)$ curve as a function of the TSR. In order to be computationally affordable, the whole curve has been produced using an intermediate resolution of $D / h=48$ mesh points or particles per diameter; it allows us to identify the optimum power operating point at TSR $=3.21$. We investigate the behaviors of the aerodynamics and wake topology of this baseline point, two off-design points (TSR $=2.14,4.28$ ), and also different aspect ratios $\mathrm{AR}=1.0,3.0$. These configurations have been simulated at a fine resolution $D / h=96$ and in domains that extended up to 17 diameters downstream of the rotor axis.

Figure 3 presents the aerodynamic behavior at mid-height. A lower-resolution result $(D / h=48)$ is also shown for the baseline TSR and demonstrates the converged state of our simulations. The aspect ratio only marginally affects the aerodynamics in the middle of the blades; its effects will be discussed further below. A positive angle of attack corresponds to a relative velocity coming from outside of the

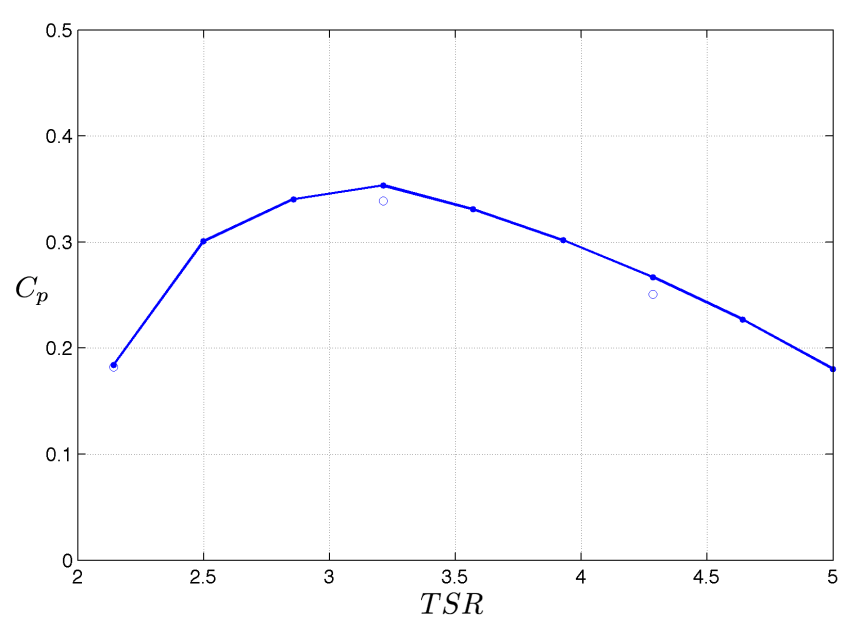

Figure 2. H-type VAWT with $\mathrm{AR}=1.5$ : power coefficient curve obtained at intermediate resolution $(D / h=48$, solid line) and configurations investigated at high resolution $(D / h=96$, circles).

cylinder swept by the blades. The angle of attack evolution during a revolution is not symmetrical for the upstream and downstream legs because of the reduced velocity encountered downstream. At the baseline TSR, it reaches a maximum just after the most upstream position $\left(15^{\circ}\right.$ around $\theta=120^{\circ}$ ) and the downstream region is characterized by a plateau close to $-7^{\circ}$. Also of note are the oscillations around 210 and $330^{\circ}$, in the angle of attack and the force coefficients. These are quite well-resolved and physical: as discussed in Sect. 3.3, the vortex sheets shed during the upstream leg do indeed impinge upon the blade in its downstream leg; the 


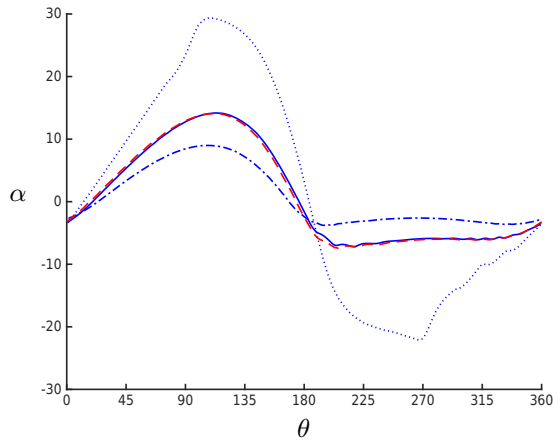

(a) Angle of attack

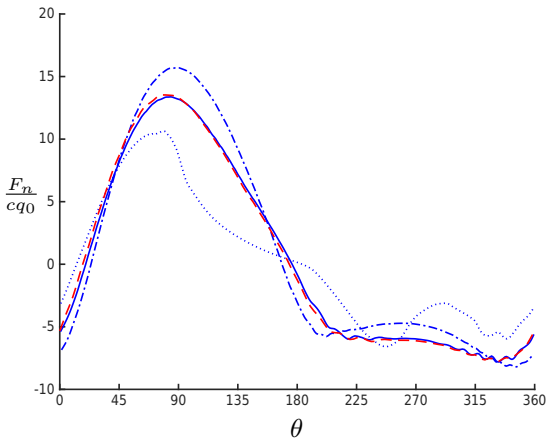

(b) Normal force coefficient

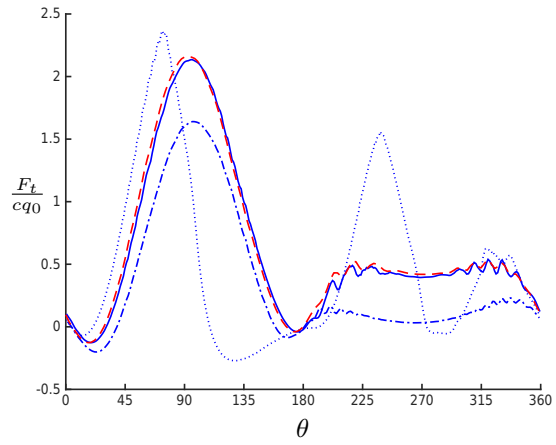

(c) Tangential force coefficient

Figure 3. H-type VAWT with $\mathrm{AR}=1.5$ : evolution of the angle of attack and of the normal, $F_{\mathrm{n}} /\left(c q_{0}\right)$, and tangential, $F_{\mathrm{t}} /\left(c q_{0}\right)$, force coefficients at mid-span versus the blade angular position $\theta$ at TSR $=2.14$ (dotted), 3.21 (solid), and 4.28 (dash-dotted); an intermediate resolution $(D / h=48)$ result for TSR $=3.21$ is also shown (dash).

Table 1. H-VAWT global performance: effects of aspect ratio, TSR, and spatial resolution.

\begin{tabular}{rr|rr|rr|rr}
\hline AR & TSR & \multicolumn{2}{|c|}{$C_{\mathrm{p}}$} & \multicolumn{2}{|c|}{$C_{x}$} & \multicolumn{2}{|c}{$C_{y}$} \\
\cline { 3 - 8 } & $D / h$ & 48 & 96 & 48 & 96 & 48 & 96 \\
\hline 1.0 & 3.21 & & 0.338 & & 0.844 & & 0.0344 \\
1.5 & 2.14 & 0.184 & 0.182 & 0.556 & 0.557 & -0.0518 & -0.0376 \\
1.5 & 3.21 & 0.353 & 0.339 & 0.863 & 0.845 & 0.0193 & 0.0435 \\
1.5 & 4.28 & 0.267 & 0.250 & 0.910 & 0.887 & 0.0390 & 0.0683 \\
3.0 & 3.21 & & 0.344 & & 0.852 & & 0.0616 \\
\hline
\end{tabular}

velocity jumps associated with these sheets then cause variations in the velocity relative to the blade.

The off-design operating points exhibit the expected behaviors: a high TSR will lead to smaller angles of attack and a decreased torque production, while the low TSR causes a distinctive stall in the upstream region and also in the downstream one. It is visible in the sharp transitions of the force coefficients at 90 and $270^{\circ}$. The angle of attack (AoA) exhibits different behaviors but is consistent with the physics. In the upstream region, the flow is dominated by the blockage effect: as the loading decreases because of stall, the AoA increases even faster; downstream, the blade initially sees a flow less impacted by the stalled upstream part but then encounters the wake of the unstalled part $\left(\theta \in\left[0,90^{\circ}\right]\right)$ and drops rapidly $\left(\theta=270^{\circ}\right)$. Finally, we summarize the effects of TSR, AR, and simulation resolution on the estimation of global performance figures in Table 1. As expected, the power, thrust, and side force coefficients are quite sensitive to the TSR. The machine aspect ratio, however, does not seem to have a major impact on them: going from $\mathrm{AR}=1$ to 3 only improves the $C_{\mathrm{p}}$ by less than $2 \%$.

\subsection{Wakes}

\subsubsection{Vortex dynamics}

The instantaneous wakes of the $\mathrm{AR}=1.5$ machine at the three considered TSRs are visualized through volume rendering of the vorticity magnitude in Fig. 4. They allow several insights into the complex vortical structure of the wake, which is significantly different from that of a HAWT. We first consider the design TSR (Fig. 4b). The vorticity shed in the wake consists of (i) the blade tip vortices, which constitute the top and bottom sides of the wake, and (ii) the vortex sheets, shed due to the time variation of the circulation of the blades, which form the lateral sides. The tip vortices are the strongest in the vicinity of the upstream- and downstream-most positions of the blades (around $\theta=90^{\circ}$ and $270^{\circ}$ ) where the blades operate at their maximum angle of attack. There, depending on the appearance of stall, or delayed stall effects, the blade will achieve its maximum circulation and then lose it either abruptly or progressively, depending on whether the blade is stalled or not. At the design TSR, the blade exploits the delayed stall to the greatest extent: its circulation keeps increasing, well past $\theta=90^{\circ}$, and then smoothly decreases. This is explained by two phenomena: (1) the airfoil experiences the highest delay in the circulation development (beneficial in this case as it widens the extent of torque production by the blade); (2) the leadingedge vortex does not introduce a sharp drop in circulation yet (which clearly happens at lower TSR, see Fig. 3b). The $\mathrm{d} \Gamma / \mathrm{d} t$ vorticity shedding is maximal when the blades are close to their lateral positions $\theta=0^{\circ}$ (upwind leg) and $180^{\circ}$ (downwind leg). The corners of the wake, i.e., the intersections of the two types of vortical structures described above, give rise to the fastest-growing vortical instabilities, which quickly propagate and cause the pairing of vortices of unequal circulations. Indeed, the unsteady aerodynamics have produced vortices with a varying circulation and the shed 


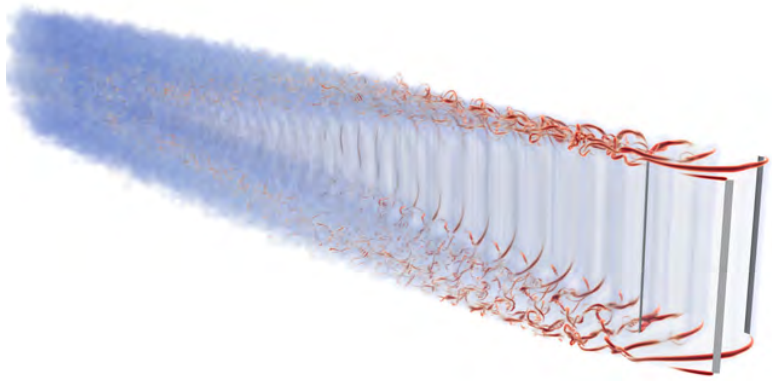

(a) $\mathrm{TSR}=2.14$

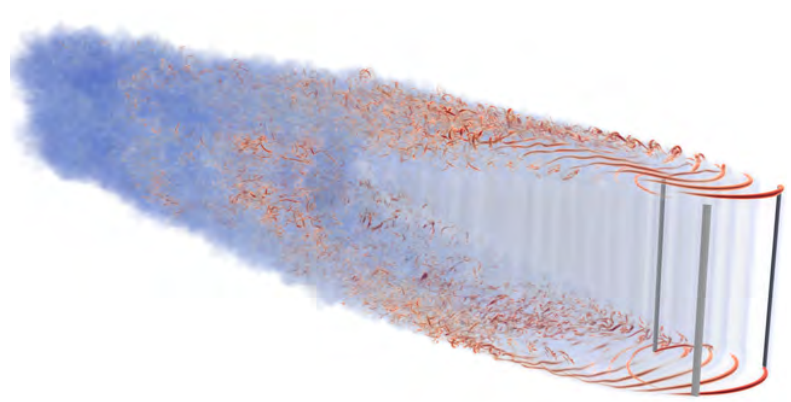

(b) $\mathrm{TSR}=3.21$

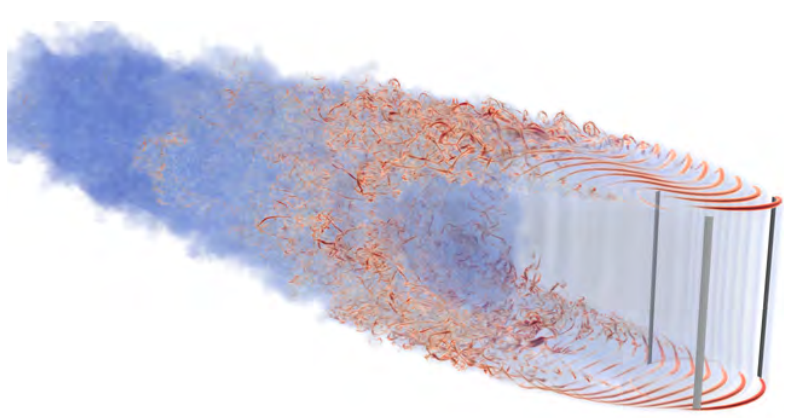

(c) $\mathrm{TSR}=4.28$

Figure 4. H-type VAWT with $\mathrm{AR}=1.5$ : volume rendering of the vorticity magnitude $\|\omega\|$; the lifting lines are also shown as 3-D blades.

vortices will interact with a different section of a preceding/succeeding vortex. In this kind of event, the stronger vortex distorts the weaker one, leading to intense stretching, enstrophy production, and the propagation of disturbances along the vortex cores, therefore bringing an overwhelming contribution to the transition to turbulence. This mechanism, most visible in Fig. 4a and isolated in Fig. 5, is well known in vortex dynamics and has already been identified on aircraft wakes (Bristol et al., 2004; Leweke et al., 2016). As a direct consequence, the turbulent regions of the wake grow from the corners and the wake only reaches a fully turbulent state once these regions have merged: the distance to reach this state will be governed directly by the aspect ratio of the machine.

The VAWT wake decay is of course also governed by the TSR in a fashion very similar to that of the HAWT: a high

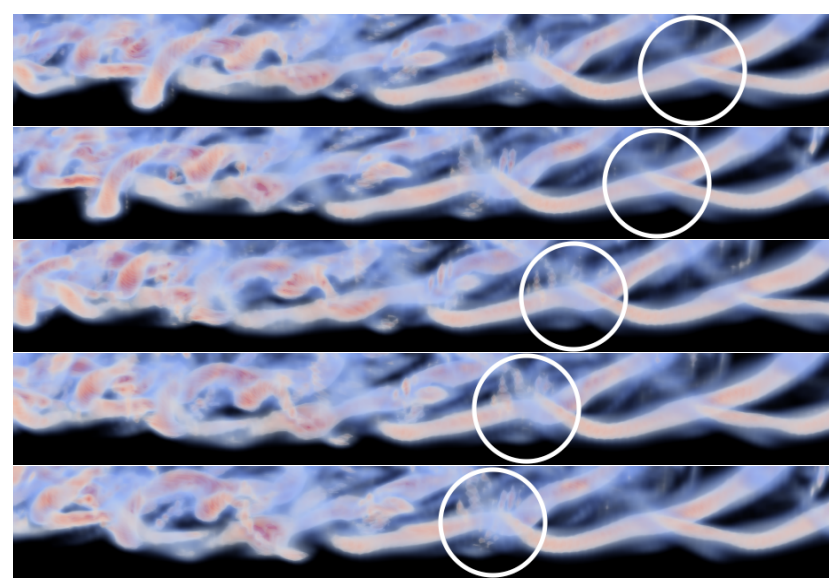

Figure 5. H-type VAWT with $\mathrm{AR}=1.5$ : volume rendering of the vorticity magnitude $\|\omega\|$; vortex reconnections are visible on the side of the wake, here illustrated in the area behind the bottom left corner of the VAWT, at successive times. The turbine is on the right, and the velocity is directed to the left.

TSR (Fig. 4c) induces narrower vortex separations, which directly condition the growth rate of the instabilities and the time to the reconnection events. This directly, and very geometrically, translates into an increasing opening angle for the envelopes of the corner vortical structures, going from Fig. 4a to c. Decreasing the TSR below the design point actually affects the wake even more dramatically. The stall event on the upstream part of the revolution weakens the upstream wake contribution (between $\theta=90$ and $180^{\circ}$ ), generating a stopping vortex that will be advected through the rotor (Fig. 4a). One can thus expect a two-lobed wake. Conversely, higher TSRs exhibit weaker vortical structures being advected through the rotor. As discussed in Sect. 3.2, the $\mathrm{d} \Gamma / \mathrm{d} t$ sheets shed on the upstream leg will cross the rotor and impact the blade aerodynamics on the downstream leg, with an extreme case being the stall event discussed above.

The behaviors of the upstream tip vortices within the rotor are more complex to apprehend, as they are affected by several factors: the intrinsic roll-up dynamics of a vortex sheet (with a time-varying strength) and the velocities induced by the surrounding vortical structures, including the bound vortices on the blades. To some degree, the latter can be crudely linked to the overall rotor loading (the $C_{x}$ values of Table 1). For a highly loaded rotor (TSR $=3.21$ and 4.28), the generated blockage effects will push the vortices shed upstream vertically and away from the downstream blade tips. One only sees the upstream tip vortices impinging upon the downstream blades at a low rotor loading, as is the case for $\mathrm{TSR}=2.14$. This observation does not agree with the results of Scheurich and Brown (Scheurich and Brown, 2013), which showed upstream vortices colliding with the blades at high TSRs. A possible explanation might lie in the relatively short domain and the direct use of the unbounded Biot- 


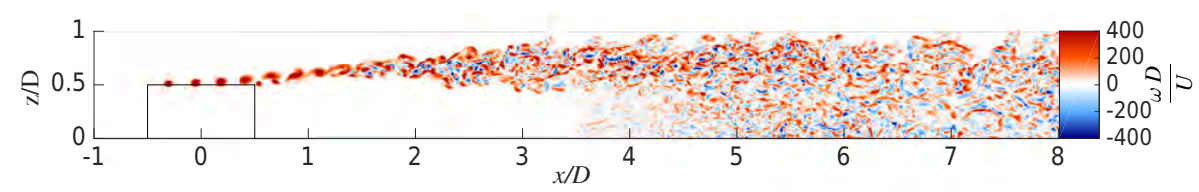

(a) $\mathrm{AR}=1.0$
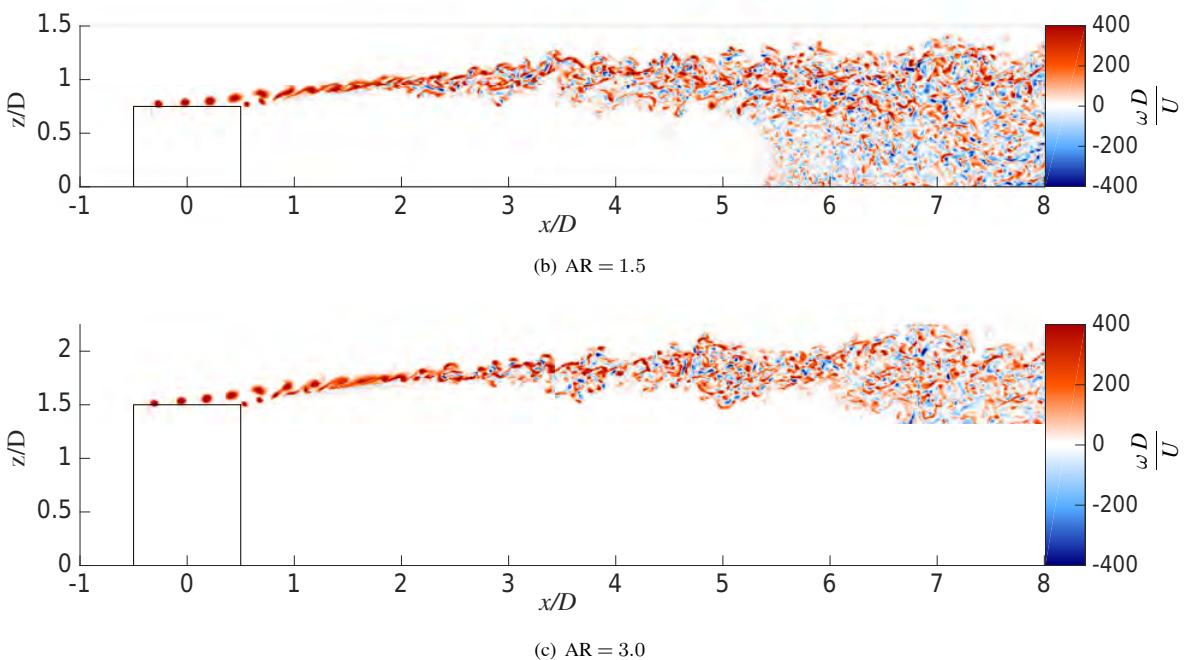

Figure 6. Effect of aspect ratio at TSR $=3.21$ : contours of the instantaneous cross-stream vorticity component $\omega_{y} D / U_{\infty}$.

Savart law in their work. One needs to add additional terms to enforce an outflow condition for this otherwise clipped vorticity field; the present study does precisely that by enforcing a normal outflow velocity $(\partial u / \partial x=0, v=0, w=0)$ through its Fourier-based solver (Chatelain and Koumoutsakos, 2010). Other explanations could also be found in a mismatch in the achieved loading by the VAWT and the use of a curvature correction; these effects should be further investigated.

Blockage is but one factor, however, and it is a global one. The discussion can be refined as additional, and less immediate, effects are to be expected from the machine geometry. The aspect ratio, as indicated by $C_{x}$ in Table 1 , has a small effect on the blockage and one can also expect an influence on the 3-D topology of this blockage effect: a higher AR thus leads to an increased clearance between the vortices and the blade, as shown in Fig. 6. The number of blades also has a strong influence; the two-bladed machine of Sect. 3.1 (not shown here; see also He, 2013) exhibits such vortex-blade collisions, in spite of its high loading $C_{x}=0.874$. Finally, beyond the rotor, the instantaneous vorticity fields of Fig. 6 also offer some insights into the pairing phenomenon of the tip vortices, the generation of a turbulent wake, and the recirculation region.

\subsubsection{Average flow statistics}

The average behavior of these wakes is studied through the mean axial velocity $\bar{u}$ and the turbulent kinetic en- ergy $\bar{k}$; these statistics were collected over a period $T_{\text {avg }}=$ $30 \mathrm{D} / U_{\infty}$. Figure 7 shows a horizontal slice of these statistics for the $\mathrm{AR}=1.5$ machine. This averaged wake exhibits several prominent features that reflect the phenomena identified in the discussion above. In all the conditions, we observe the generation of turbulent kinetic energy (TKE) on the sides of the wake and the associated smearing of the velocity deficit. This is consistent with our discussion of the vortical instabilities in the corner structures and the subsequent propagation of the turbulent regions. At low TSR, the averaged velocity field exhibits the expected two-lobed structure, with a stronger deficit on the side of the rotor on which the blade is traveling upwind $\left(\theta \in\left[270,90^{\circ}\right]\right)$. For the higher TSRs (Fig. 7b and c), a backflow region lies inside the wake at a position that varies with the TSR: it is centered at $x / D \simeq 5.5$ for $\mathrm{TSR}=3.21$ and at $x / D \simeq 4$ for $\mathrm{TSR}=4.28$. The location of this feature clearly coincides with the production of TKE and an accelerated smearing of the wake velocity deficit; this too agrees with our vortex dynamics discussion. The topology of the associated recirculation bubbles is clearly threedimensional and will not be discussed here.

Finally, the averaged wakes exhibit a slight deviation in this midplane. As expected, the behaviors of the three TSRs do correlate with the signs and values of the side forces produced by the rotor (see $C_{y}$ in Table 1). These side forces also appear in the average behavior as observed in cross-flow slices (Fig. 8). The deformation of the velocity deficit clearly suggests the presence of mean streamwise vortices along the 


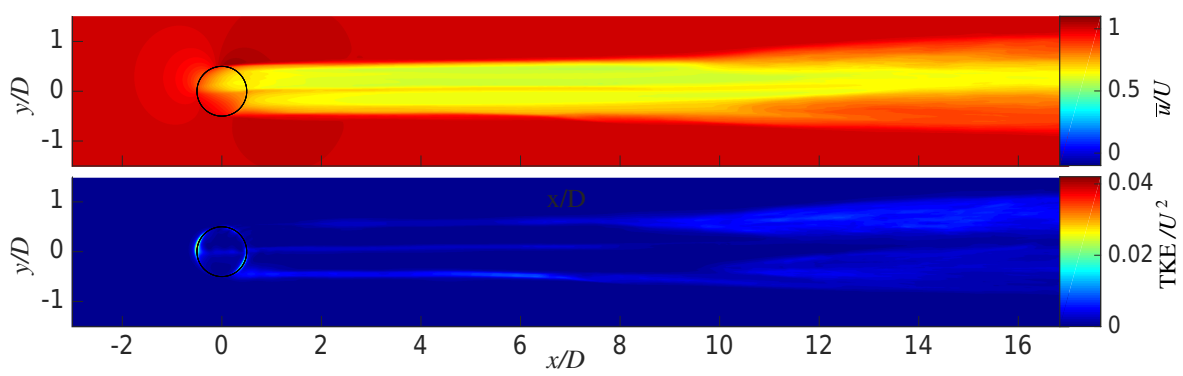

(a) $\mathrm{TSR}=2.14$

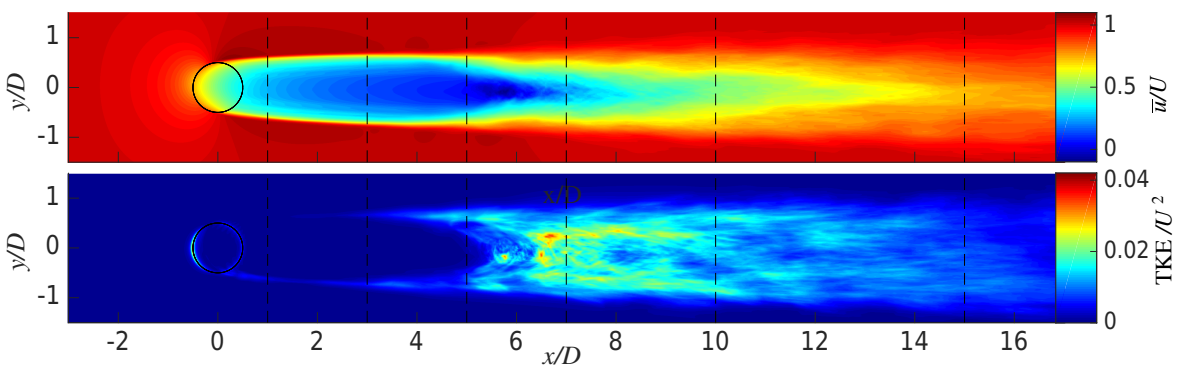

(b) $\operatorname{TSR}=3.21$

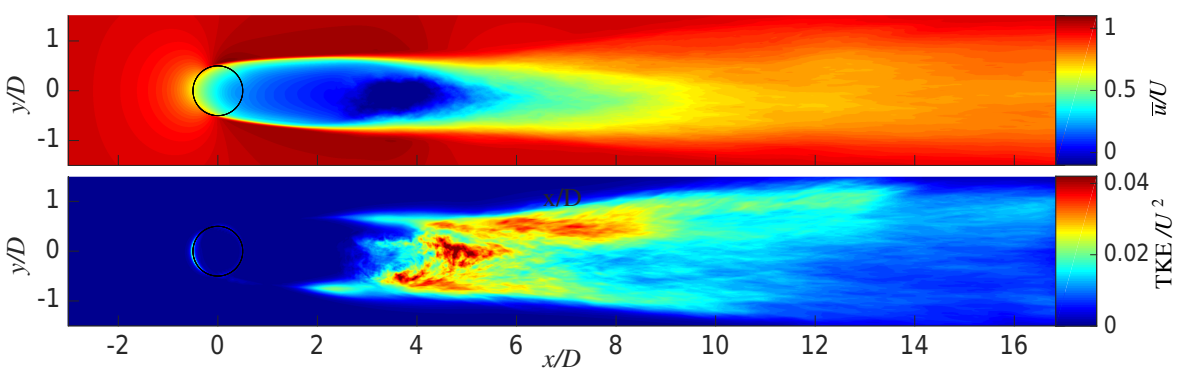

(c) $\mathrm{TSR}=4.28$

Figure 7. H-type VAWT with $\mathrm{AR}=1.5$ : mean streamwise velocity $\bar{u} / U_{\infty}$ and resolved turbulent kinetic energy $\bar{k}=\frac{\overline{u^{\prime} u^{\prime}}+\overline{v^{\prime} v^{\prime}}+\overline{w^{\prime} w^{\prime}}}{2 U_{\infty}^{2}}$ in the $y / D=0$ plane.

corners of the wake (Fig. 8a and b), a clear departure from a HAWT wake with no side slip angle.

The mean streamwise vorticity at three transverse slices is shown in Fig. 9. Even though the statistics are converged, the near-perfect periodicity of the flow leads to a pattern of positive and negative patches, signatures of the advection of tip vortices shed on the upstream and downstream parts of the rotation, respectively. The dominant streamwise vorticity is thus difficult to identify in the near-wake, but large-scale structures can be identified further downstream, also thanks to the induced deformation of the wake.

\subsubsection{Decay diagnostics}

We apply classical turbulent wake diagnostics to the characterization of the wake decay. More specifically, we adapt integral quantities, such as the displacement and momentum widths, to the present context; the wakes considered do in- deed lack symmetry and exhibit strong secondary flow structures, which makes the definition of a velocity deficit evolution based upon a single characteristic point unsuitable. Thus, we define dimensionless displacement and momentum surfaces, respectively, as

$$
\begin{aligned}
S_{1}(x)= & \frac{1}{H D} \int_{-\infty}^{\infty} \int_{-\infty}^{\infty}\left(1-\frac{u_{x}(x, y, z)}{U_{\infty}}\right) \mathrm{d} y \mathrm{~d} z \\
S_{2}(x)= & \frac{2}{H D} \int_{-\infty}^{\infty} \int_{-\infty}^{\infty}\left(1-\frac{u_{x}(x, y, z)}{U_{\infty}}\right) \\
& \left(\frac{u_{x}(x, y, z)}{U_{\infty}}\right) \mathrm{d} y \mathrm{~d} z .
\end{aligned}
$$

These diagnostics correspond to integrals of flux quantities in cross-stream sections located at a distance $x$ downstream of the turbine axis; their practical implementation approxi- 


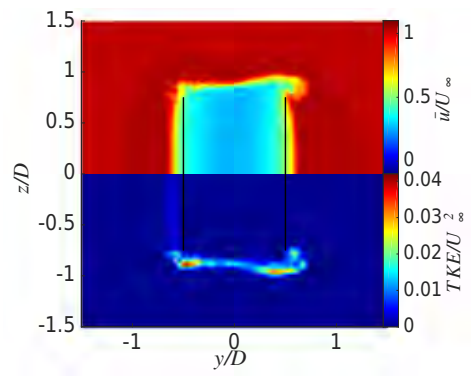

(a) $x / D=1$

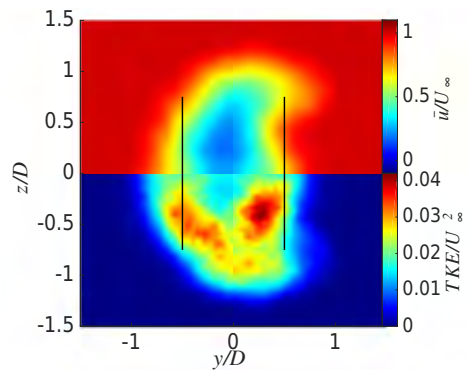

(d) $x / D=7$

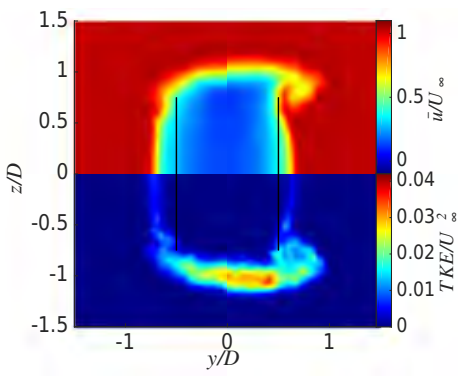

(b) $x / D=3$

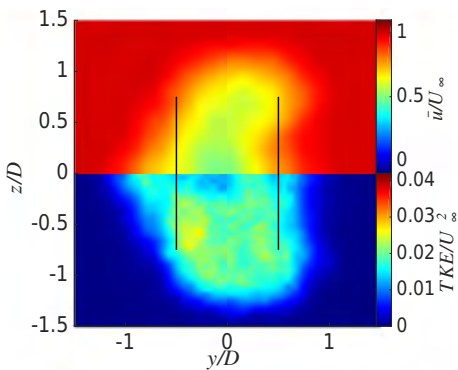

(e) $x / D=10$

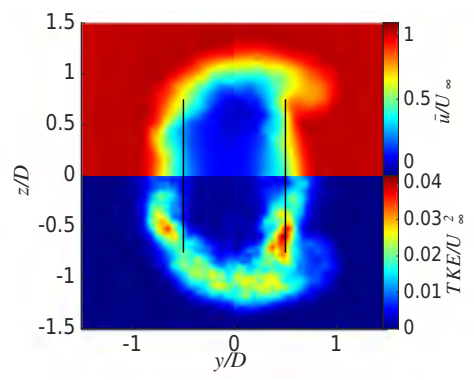

(c) $x / D=5$

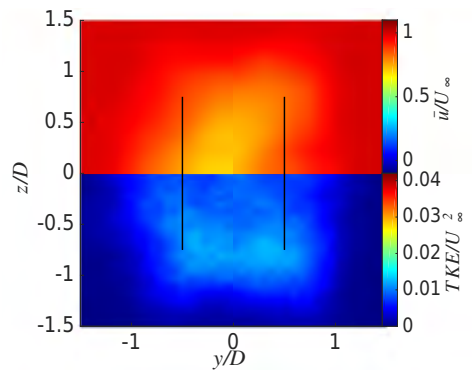

(f) $x / D=15$

Figure 8. H-type VAWT with $\mathrm{AR}=1.5$ : mean streamwise velocity $\bar{u} / U_{\infty}$ and turbulent kinetic energy $\bar{k}$ in cross-flow slices.

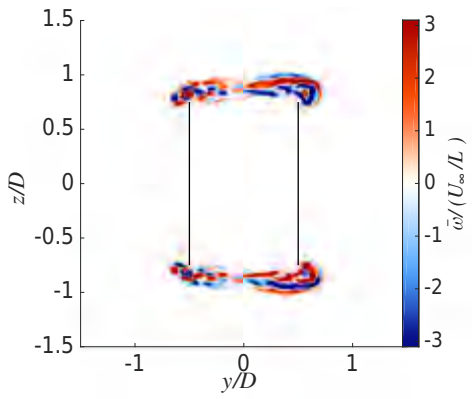

(a) $x / D=1$

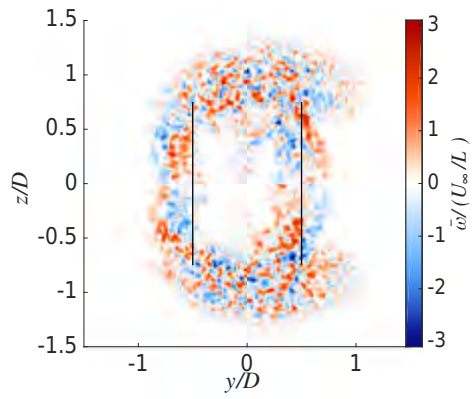

(b) $x / D=5$

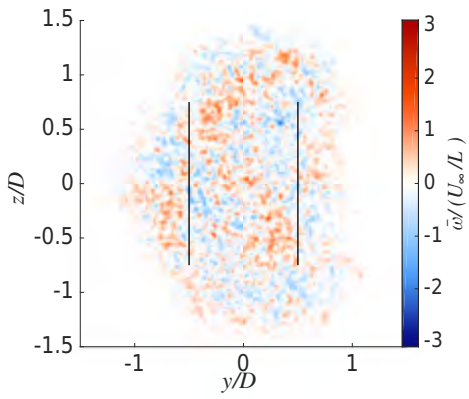

(c) $x / D=10$

Figure 9. H-type VAWT with $\mathrm{AR}=1.5$ : mean streamwise vorticity $\bar{\omega} /\left(U_{\infty} / L\right)$.

mates these integrals through quadrature over finite square sections $[-3 D, 3 D] \times[-3 D, 3 D]$. Because our Biot-Savart solver enforces transverse unbounded conditions exactly, it allows a transverse mass flow due to blockage. As a consequence, $S_{1}$, shown in Fig. 10a, does not vanish (as it would have for a solver with no through-flow boundaries); it quantifies the blockage effect caused by the wake on the flow. As a reference, $S_{1}$ should be compared with the square of the displacement width $\left(\delta^{2}\right)$ of an axisymmetric wake for which classical similarity theory (Tennekes and Lumley, 1972) predicts a behavior $S_{1} \sim x^{-1 / 3}$ in the far wake. The asymmetry of the wake generator and its proximity are such that we cannot observe the self-similarity region: classical results for bluff bodies indicate a development distance of $x / D \sim 50$ to obtain the theoretical far-wake self-similarity (Pope, 2001). The decay observed for $x / D>5$ for most of the configurations does, however, suggest a power-law-like behavior. For HAWTs, it has been observed that the decay deviates significantly from the bluff body behavior in the presence of a turbulence inflow (Litvinov et al., 2015); similarly, it will be interesting to assess the sensitivity of VAWT wake decay with respect to the turbulence intensity. Still, the evolution of $S_{1}$ does provide a signature of the recirculation region: the magnitude and the extent of the overshoot $S_{1}>1$ correlate with the location and the size of the recirculation bubble for the design and high TSRs (Fig. 7b and c). This correspondence 


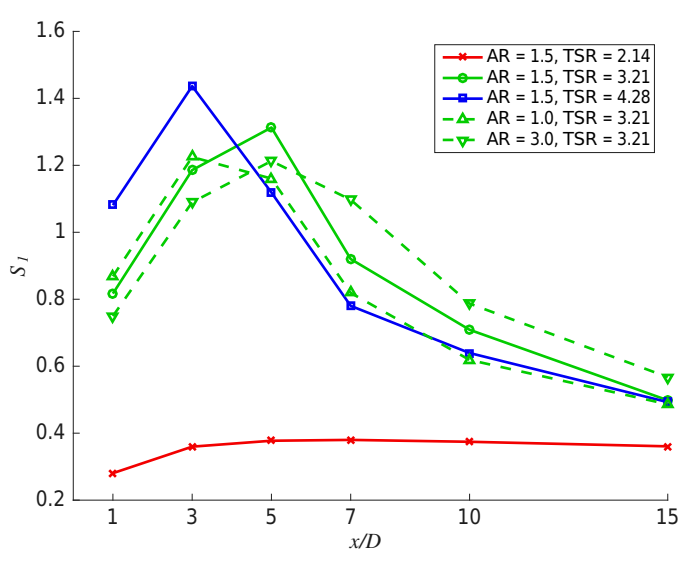

(a) Dimensionless displacement surface $S_{1}$

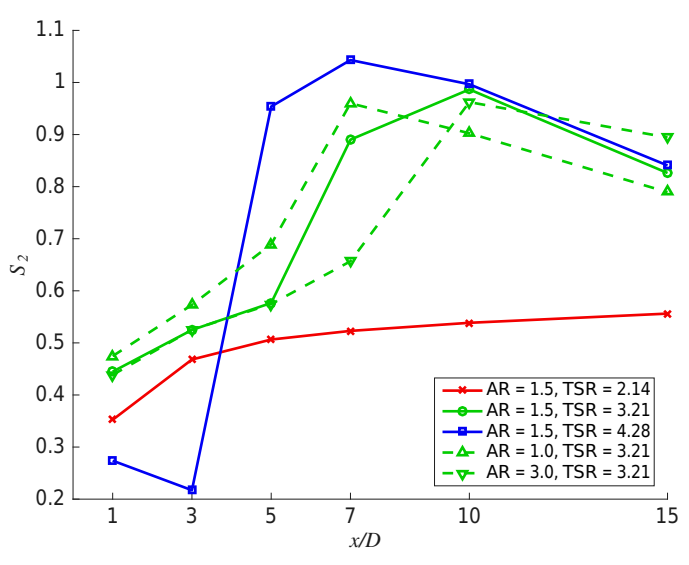

(b) Dimensionless momentum surface $S_{2}$

Figure 10. H-type VAWT: dimensionless displacement and momentum surfaces as functions of the streamwise coordinate.

also agrees with the effect of the aspect ratio: an increasing AR pushes both the recirculation (indicated by the merging of vortical structures in the center of the wake in Fig. 6) and the $S_{1}$ overshoot further downstream.

The dimensionless momentum surface $S_{2}$ is related to the deficit in the flux of momentum in these planes. In the absence of secondary flows and pressure gradients, it should in fact correspond to the thrust coefficient $S_{2} \simeq C_{x}$ at large distances behind the VAWT when a factor 2 is used in the definition of $S_{2}$, as here in Eq. (9). This is confirmed by our results of Fig. 10b: after a transition, the curves tend towards the corresponding $C_{x}$ values of Table 1.

Finally, the case TSR $=2.14$ constitutes an outlier in the discussions above. This is not unexpected: the instability growth is slower than for the other cases and does not allow the transition to a well-mixed fully turbulent wake within the computational domain.

\section{Conclusions}

A vortex particle-mesh method, here briefly presented, has been applied to large-scale and high-resolution large-eddy simulation (LES) of VAWT wakes. The method is capable of tracking vortical structures over very long times and distances. This has led to several insights into the vortex dynamics at work inside the wakes of VAWTs. The mean flow topology has been extracted; unsteady flow aspects, threedimensional effects and classical wake diagnostics have also been studied. The impact of several of these flow features for the deployment of VAWTs in wind farms is considerable: the aspect ratio and the operating conditions of the machine greatly affect the wake decay and even allow the presence of a recirculation region. The present study merely constitutes a preliminary study of VAWT wakes. Direct follow-up work will investigate the 3-D topology of the averaged wake and its unsteadiness. We will then also consider the behavior of these machines and of their wakes in a turbulent wind. Our methodology can also accommodate rotor dynamics models and realistic controllers; this will bring definitive answers to the smoothness of torque generation for H-type VAWTs and their performances in wind farms.

Code availability. The immersed lifting line VPM code and its Fourier-based solver library are proprietary. The Parallel ParticleMesh (PPM) library is an open-source library (ETHZ/CSE Lab, 2011).

Data availability. The data sets involved in this study consist of massive 3-D and time-dependent data sets, the handling of which is not tractable on a data registry. Readers interested in the raw simulation data or the post-processed statistics are invited to contact the authors.

\section{The Supplement related to this article is available online at https://doi.org/10.5194/wes-2-317-2017-supplement.}

Author contributions. PC and MD prepared and ran the simulations, and DGC performed their post-processing. PC and MD developed the code; YM and DGC developed the dynamic stall model inside the code. PC, MD, and GW contributed to the analysis and the discussion of the results. PC prepared the paper with contributions from all co-authors.

Competing interests. The authors declare that they have no conflict of interest.

Acknowledgements. The authors acknowledge the fruitful discussions with Thierry Maeder, Stefan Kern, and Dominic 
von Terzi at the Aerodynamics and Acoustic Lab at GE Global Research, Garching bei München. Matthieu Duponcheel was partially supported by the ENGIE-funded research project Small Wind Turbines. The development work benefited from the computational resources provided by the supercomputing facilities of the Université catholique de Louvain (CISM/UCL) and the Consortium des Équipements de Calcul Intensif (CÉCI) en Fédération Wallonie Bruxelles (FWB) funded by the Fond de la Recherche Scientifique de Belgique (F.R.S.-FNRS) under convention no. 2.5020.11. The production simulations used computational resources made available on the Tier-1 supercomputer of the FWB, infrastructure funded by the Walloon Region under grant agreement no. 1117545 .

Edited by: Carlo L. Bottasso

Reviewed by: two anonymous referees

\section{References}

Beaudet, L.: Etude expérimentale et numérique du décrochage dynamique sur une éolienne à axe vertical de forte solidité, $\mathrm{PhD}$ thesis, Université de Poitiers, 2014.

Bristol, R. L., Ortega, J. M., Marcus, P. S., and Savas, O.: On cooperative instabilities of parallel vortex pairs, J. Fluid Mech., 517, 331-358, 2004.

Brown, R. E. and Line, A.: Efficient High-Resolution Wake Modeling Using the Vorticity Transport Equation, AIAA J., 43, 1434 1443, 2002

Castelein, D.: Dynamic stall on vertical Axis Wind Turbines - Creating a benchmark of Vertical Axis Wind Turbines in Dynamic Stall for validating numerical models, Master's thesis, Technische Universiteit Delft, 2015.

Chatelain, P. and Koumoutsakos, P.: A Fourier-based elliptic solver for vortical flows with periodic and unbounded directions, J. Comput. Phys., 229, 2425-2431, http: //www.sciencedirect.com/science/article/B6WHY-4Y4R4GD-1/ 2/1cf355678ffad889ad409f181e47840d, 2010.

Chatelain, P., Curioni, A., Bergdorf, M., Rossinelli, D., Andreoni, W., and Koumoutsakos, P.: Billion vortex particle Direct Numerical Simulations of aircraft wakes, Comput. Method. Appl. M., 197, 1296-1304, 2008.

Chatelain, P., Backaert, S., Winckelmans, G., and Kern, S.: Large Eddy Simulation of wind turbine wakes, in: Proceedings of The 9th International Symposium on Engineering Turbulence Modelling and Measurements (ETMM-9), June 6-8, 2012, Thessaloniki, Greece, edited by: Rodi, W., vol. 91 of Flow, Turbulence and Combustion, 587-605, ERCOFTAC, Springer, https://doi.org/10.1007/s10494-013-9474-8, 2013.

Cocle, R., Dufresne, L., and Winckelmans, G.: Investigation of multiscale subgrid models for LES of instabilities and turbulence in wake vortex systems, Lect. Notes Comp. Sci., 56, 141-159, 2007

Cocle, R., Winckelmans, G., and Daeninck, G.: Combining the vortex-in-cell and parallel fast multipole methods for efficient domain decomposition simulations, J. Comput. Phys., 227, 9091-9120, https://doi.org/10.1016/j.jcp.2007.10.010, 2008.

Cocle, R., Bricteux, L., and Winckelmans, G.: Scale dependence and asymptotic very high Reynolds number spectral behavior of multiscale subgrid models, Phys. Fluids, 21, 085101, https://doi.org/10.1063/1.3194302, 2009.
Cottet, G.-H.: Artificial viscosity models for vortex and particle methods, J. Comput. Phys., 127, 299-308, 1996.

Dyachuk, E., Goude, A., and Bernhoff, H.: Dynamic stall modeling for the conditions of vertical axis wind turbines, AIAA J., 52, 72-81, 2014.

ETHZ/CSE Lab: http://www.ppm-library.org (15 June 2017), 2011.

Ferreira, C. S., van Kuik, G., van Bussel, G., and Scarano, F.: Visualization by PIV of dynamic stall on a vertical axis wind turbine, Exp. Fluids, 46, 97-108, https://doi.org/10.1007/s00348008-0543-z, 2009.

Ferreira, C. S., Madsen, H. A., Barone, M., Roscher, B., Deglaire, P., and Arduin, I.: Comparison of aerodynamic models for Vertical Axis Wind Turbines, J. Phys. Conf. Ser., 524, 012125, http://stacks.iop.org/1742-6596/524/i=1/a=012125, 2014.

He, C.: Wake Dynamics Study of an H-type Vertical Axis Wind Turbine, Master's thesis, Technische Universiteit Delft, 2013.

Hockney, R. and Eastwood, J.: Computer Simulation using Particles, Taylor \& Francis, Inc. Bristol, PA, USA, 1988.

Hughes, T. J. R., Mazzei, L., Oberai, A. A., and Wray, A. A.: The multiscale formulation of large eddy simulation: Decay of homogeneous isotropic turbulence, Phys. Fluids, 13, 505-512, https://doi.org/10.1063/1.1332391, 2001.

Jeanmart, H. and Winckelmans, G.: Investigation of eddy-viscosity models modified using discrete filters: A simplified "regularized variational multiscale model" and an "enhanced field model", Phys. Fluids, 19, 055110, https://doi.org/10.1063/1.2728935, 2007.

Kinzel, M., Mulligan, Q., and Dabiri, J. O.: Energy exchange in an array of vertical-axis wind turbines, J. Turbul., 13, N38, https://doi.org/10.1080/14685248.2012.712698, 2012.

Koumoutsakos, P.: Inviscid axisymmetrization of an elliptical vortex, J. Comput. Phys., 138, 821-857, https://doi.org/10.1006/jcph.1997.5749, 1997.

Koumoutsakos, P.: Multiscale flow simulations using particles, Annu. Rev. Fluid Mech., 37, 457-487, 2005.

Leishman, J. G.: Principles of helicopter aerodynamics, Cambridge University Press, 2nd edn., 2006.

Leweke, T., Le Dizès, S., and Williamson, C. H. K.: Dynamics and Instabilities of Vortex Pairs, Annu. Rev. Fluid Mech., 48 507-541, https://doi.org/10.1146/annurev-fluid-122414-034558, 2016.

Litvinov, I., Naumov, I., Okulov, V., and Mikkelsen, R. F.: Comparison of far wakes behind a solid disk and a three-blade rotor, Journal of Flow Visualization and Image Processing, 22, 175-183, https://doi.org/10.1615/JFlowVisImageProc.2016016609, 2015.

Migliore, P. and Wolfe, W.: Some effects of flow curvature on the performance of Darrieus wind turbines, American Institute of Aeronautics and Astronautics, https://doi.org/10.2514/6.1979$112,1979$.

Monaghan, J.: SPH without a Tensile Instability, J. Comput. Phys., 159, 290-311, 2000.

Monaghan, J. J.: Extrapolating B splines for interpolation, J. Comput. Phys., 60, 253-262, http://www. sciencedirect.com/science/article/B6WHY-4DD1WC8-19H/ 2/5388f2c6dbe3898de31ea0ea7bbb03fa, 1985.

Paquette, J. and Barone, M. F.: Innovative Offshore Vertical-Axis Wind Turbine Rotor Project, in: EWEA 2012, 16-19 April, 2012 in Copenhagen, Denmark, EWEA, 2012. 
Ploumhans, P. and Winckelmans, G. S.: Vortex Methods for High-Resolution Simulations of Viscous Flow Past Bluff Bodies of General Geometry, J. Comput. Phys., 165, 354-406, http://www.sciencedirect.com/science/article/ B6WHY-45FC88K-3/2/3f55200e6caead3c52ecc8328dfbada8, 2000.

Pope, S. B.: Turbulent flows, IOP Publishing, 2001.

Prandtl, L.: Application of Modern Hydrodynamics to Aerodynamics, Technical Report 116, NACA, 1923.

Scheurich, F.: Modelling the aerodynamics of vertical-axis wind turbines, PhD thesis, University of Glasgow, 2011.

Scheurich, F. and Brown, R. E.: Modelling the aerodynamics of vertical-axis wind turbines in unsteady wind conditions, Wind Energy, 16, 91-107, https://doi.org/10.1002/we.532, 2013.

Scheurich, F., Fletcher, T. M., and Brown, R. E.: The Influence of Blade Curvature and Helical Blade Twist on the Performance of a Vertical-Axis Wind Turbine, in: 48th AIAA Aerospace Sciences Meeting Including the New Horizons Forum and Aerospace Exposition, AIAA 2010-1579, AIAA, 2010.
Sørensen, J. N., Mikkelsen, R. F., Henningson, D. S., Ivanell, S., Sarmast, S., and Andersen, S. J.: Simulation of wind turbine wakes using the actuator line technique, P. Roy. Soc. Lond. A Mat., 373, 20140071, https://doi.org/10.1098/rsta.2014.0071, 2015.

Tennekes, H. and Lumley, J. L.: A First Course in Turbulence, MIT Press, https://books.google.be/books?id=h4coCj-lNOcC, 1972.

van Rees, W. M., Leonard, A., Pullin, D. I., and Koumoutsakos, P.: A comparison of vortex and pseudo-spectral methods for the simulation of periodic vortical flows at high Reynolds numbers, J. Comput. Phys., 230, 2794-2805, 2011.

Williamson, J. H.: Low-storage Runge-Kutta schemes, J. Comput. Phys., 35, 48-56, http://www.sciencedirect. com/science/article/B6WHY-4DD1NRP-H1/2/ a1a09857d87c5a3538d4800fd648be47, 1980.

Winckelmans, G.: Vortex Methods, in: Encyclopedia of Computational Mechanics, edited by: Stein, E., De Borst, R., and Hughes, T. J., vol. 3, John Wiley and Sons, 2004. 